Original paper

\title{
Application of an independent dose calculation software for estimating the impact of inter-fractional setup shifts in Helical Tomotherapy treatments
}

\author{
Erik Dzintars ${ }^{\mathrm{a}}$, Nikos Papanikolaou ${ }^{\mathrm{a}}$, Panayiotis Mavroidis ${ }^{\mathrm{a}, \mathrm{b}, *}$, Amir Sadeghi $^{\mathrm{a}}$, \\ Sotirios Stathakis ${ }^{\text {a }}$ \\ ${ }^{a}$ Department of Radiation Oncology, University of Texas Health Science Center, San Antonio, TX, USA \\ ${ }^{\mathrm{b}}$ Department of Medical Radiation Physics, Karolinska Institutet and Stockholm University, Sweden
}

\section{A R T I C L E I N F O}

\section{Article history:}

Received 8 January 2012

Received in revised form

4 September 2012

Accepted 10 September 2012

Available online 6 October 2012

\section{Keywords:}

Helical Tomotherapy

Independent point dose calculation

Patient setup

Inter-fractional shifts

\begin{abstract}
A B S T R A C T
The purpose of this study is to validate the capability of in-house independent point dose calculation software to be used as a second check for Helical Tomotherapy treatment plans. The software performed its calculations in homogenous conditions (using the Cheese phantom, which is a cylindrical phantom with radius $15 \mathrm{~cm}$ and length $18 \mathrm{~cm}$ ) using a factor-based algorithm. Fifty patients, who were treated for pelvic (10), prostate (14), lung (10), head \& neck (12) and brain (4) cancers, were used. Based on the individual patient kVCT images and the pretreatment MVCT images for each treatment fraction, the corresponding daily patient setup shifts in the IEC-X, IEC-Y, and IEC-Z directions were registered. For each patient, the registered fractional setup shifts were grouped into systematic and random shifts. The average systematic dosimetric variations showed small dose deviation for the different cancer types (1.0\%-3.0\%) compared to the planned dose. Of the fifty patients, only three had percent differences larger than $5 \%$. The average random dosimetric variations showed relatively small dose deviations $(0.2 \%-1.1 \%)$ compared to the planned dose. None of the patients had percent differences larger than $5 \%$. By examining the individual fractions of each patient, it is observed that only in 31 out of 1358 fractions the percent differences exceeded the border of $5 \%$. These results indicate that the overall dosimetric impact from systematic and random variations is small and that the software is a capable platform for independent point dose validation for the Helical Tomotherapy modality.
\end{abstract}

(c) 2012 Associazione Italiana di Fisica Medica. Published by Elsevier Ltd. All rights reserved.

\section{Introduction}

The necessity of image guided radiation therapy (IGRT) for eliminating eventual geometric uncertainties has become widely accepted [1-4]. That is because the dose distribution that the modern radiation modalities produce are characterized by high conformalities, which require high precision and accuracy during in target localization during treatment planning and delivery.

On-board megavoltage computed tomography (MVCT) has enabled Helical Tomotherapy (HT) (TomoTherapy, Inc., Madison, WI, USA) to deliver very conformal dose distributions with high precision [5]. The management of the involved geometric uncertainties and their dosimetric impact during treatment delivery is of great interest. In clinical practice, these geometric uncertainties

\footnotetext{
* Corresponding author. Department of Medical Radiation Physics, Karolinska Institutet and Stockholm University, Box 260, 17176 Stockholm, Sweden.

E-mail address: panayiotis.mavroidis@ki.se (P. Mavroidis).
}

mainly stem from beam to patient misalignment, intra-fraction and inter-fraction organ or patient motion. The impact of these uncertainties in dosimetric as well as in tissue response terms is of major concern [6-8]. Different studies that have investigated the dosimetric effects of beam to patient misalignment have shown that this source of error may introduce significant discrepancies between treatment plan and dose delivery, which may have a significant effect in the expected treatment outcome [9-11].

In HT, to account for the effects of intra-fraction motion, real time motion adaptive plan optimization and treatment delivery are needed $[12,13]$. To account for the effects of inter-fraction motion, the MVCT has been proved to be a powerful tool in the daily registration of patient setup. The MVCT images can be compared with the kilovoltage computed tomography (kVCT) images of the patient, which were used during treatment planning, and eventual discrepancies between the two sets of images can be corrected in every fraction. In HT, there have been studies reporting on the effect of patient setup shifts, mainly focusing on the MVCT utility and the relevant registration strategies applied [14-20]. However, a few only studies have 
reported findings on the dosimetric and radiobiological consequences patient setup shifts in conjunction with the MVCT [21-24].

The American Association of Physicists in Medicine (AAPM) Task Group 40 recommends that the dose calculated in patient treatment plans be independently confirmed by a second check [25]. With the ever increasing advancement of technology comes an equally increasing complexity for second check calculations. Currently, verification of tomotherapy treatment plans is typically achieved through dose measurements on a clinical measurement phantom. While several commercial software products are currently available for IMRT delivery techniques, they are not yet available for the helical Tomotherapy modality.

In the literature, there are reports on approaches that perform independent dose calculation for IMRT verification, which are based on very sophisticated algorithms $[26,27]$. For example, an effective method for model-based dose calculations is offered by combining multi-source modeling of the energy fluence exiting the treatment head with use of energy deposition kernels describing the energy deposition in the patient through convolution/superposition with the energy fluence incident on the irradiated object.

Software solutions implementing Monte Carlo models for intensity modulated sequential tomotherapy (IMST), stereotactic body radiotherapy (SBRT), three-dimensional conformal radiotherapy, and intensity modulated radiotherapy (IMRT), as well as a recent algorithm for point dose calculation in Helical Tomotherapy have all been used for dose calculation [28-32]. Independent dose calculation software exists for brachytherapy, Cyberknife, conventional, and IMRT treatment modalities while the Helical Tomotherapy modality is not currently supported in the same capacity. We assess a preliminary version of an in-house software tool designed to translate and write Helical Tomotherapy treatment information into a non-helical format such that an independent dose calculation method implementing the EGS4/MCSIM software environment is viable.

The goal of this study is to validate an independent point dose calculation software for the HT treatment planning system in order to investigate the dosimetric consequence of inter-fractional patient setup shifts using the HT treatment plan and delivery data of fifty cancer patients of various cancer types. In this work, as systematic patient setup shift is defined the mean daily patient setup shift, whereas as random patient setup shift is defined the fractional patient setup shift where the corresponding systematic patient setup shift has been subtracted. Both the systematic and random patient setup shifts were introduced into the independent point dose calculation software, to evaluate the dosimetric impact of the corresponding systematic and random patient setup variations.

\section{Materials and methods}

In this study, fifty cancer patients from multiple treatment sites, who were treated by Helical Tomotherapy, were randomly selected. Of the total group of patients, ten were treated for pelvic, fourteen for prostate, ten for lung, twelve for head \& neck and four for brain cancers, respectively. For each patient, the kVCT images, which were acquired by a GE light speed 4-slice CT scanner (General Electric Medical Systems, Milwaukee, WI, USA), and the corresponding treatment plan were available. For each patient, the kVCT images were imported to the Pinnacle ${ }^{3}$ treatment planning system (Pinnacle ${ }^{3}$ TPS, Philips Medical Systems, Andover, MA, USA) and the planning target volume (PTV) and organs at risk(OAR) were delineated. Subsequently, the contoured kVCT images were transferred to the HT treatment planning station using the DICOM protocol and the corresponding treatment plan was produced using the $2.5 \mathrm{~cm}$ jaw setting.

The individual patient treatment plans were optimized using the treatment constraints of the clinical protocol. The final treatment configuration was applied during treatment delivery and its accuracy was validated through the production of delivery quality assurance (DQA) dose distributions. These DQA dose distributions were produced using the Cheese phantom (a cylindrical phantom with radius $15 \mathrm{~cm}$ and length $18 \mathrm{~cm}$ ). A random point inside the tumor was selected for comparing the point dose that was provided by the treatment planning system with the point dose at the same point in the Cheese phantom that was provided by an independent dose calculation software. The reason for considering only one point is because the authors wanted to validated the software as a clinical tool, which could verify the reproducibility of the reference dose and in this way to estimate the change (if any) to which the whole dose distribution has been subjected.

Based on the individual patient kVCT images and the pretreatment MVCT images for each treatment fraction, the corresponding daily patient setup shifts in the IEC-X, IEC-Y, and IEC-Z directions were obtained and quantified. The registered inter-fractional daily setup shifts together with the selected point coordinates were imported in the DQA treatment configuration and the independent dose calculation software recalculated the dose to examine the dosimetric impact due to the relevant inter-fractional patient setup shifts. Due to the fact that previous studies showed that the relevant dosimetric discrepancies for gantry rotation were negligible $[9,33]$, the rotational effects were not examined in this study.

\section{Independent point dose calculation for Helical Tomotherapy}

The ability to calculate a point dose is one of the basic techniques used for independent dose calculation. A point dose calculated by the treatment planning system can be verified through a chamber measurement or with independent dose verification. In a recent study, Papanikolaou et al. [28] developed a calculation algorithm to "confirm the dose to a point in a high dose, low dosegradient region" for helical Tomotherapy. The algorithm incorporates patient specific data, common dosimetric functions, and machine data in its point dose calculation [34].

The calculations of the in-house software are based on the cylindrical 'Cheese' phantom (TomoTherapy Inc., Madison, WI), commonly used for Helical Tomotherapy QA. This same phantom is used to create DQA plans in the treatment planning system. Geometric calculations determine the depth in the 'Cheese' phantom along the different projections. The present algorithm was applied to four different phantoms (two homogeneous and two heterogeneous) and it was found that the agreement between the treatment planning calculations and those provided by the independent point dose calculations was $1-2 \%$ [28].

\section{Dose calculation: inter-fractional shifts}

In HT treatments, shifts can be applied in each fraction to correct for proper patient alignment. Tomotherapy treatment plans are based on kVCT images, which are stored in the treatment station. Prior to treatment, MVCT scans are registered against these original kVCT images and shifts are introduced to correct for eventual patient positioning. Shifts can be in the lateral (IEC-X), superior-inferior (IEC-Y), anterior-posterior (IEC-Z), and rotational directions. The implemented shifts are recorded in each treatment fraction.

The in-house software was applied to each patient to investigate how inter-fractional shifts affect dose delivery, and therefore the use of an independent dose calculation, becomes an important issue. Independent point dose calculations were performed in each patient with default, systematic, and random shift input values.

The first independent dose calculations were performed using the default, original point of measurement input. The point of measurement input is defined by the relative differences between 
the green and red laser points, and it is the default position for the in-house software. This provides a standard point dose calculation with no inter-fractional shift correction.

The second round of independent dose calculations involves a systematic shift input. For each patient in this study, raw setup shifts were recorded prior to each treated fraction. The average of those recorded fractional shifts defines the associated patient specific systematic shift. This value defines the average setup error observed during the course of the tomotherapy treatment. The systematic shift values are applied to the IEC-X, IEC-Y, and IEC-Z coordinates for the default point of measurement, resulting in a shift-corrected point of measurement. This independent point dose calculation represents the average dosimetric effect due to inter-fractional shifts.

Finally, independent point dose calculations are computed for each treatment fraction. Subtracting the associated systematic shift values from the raw recorded fractional setup shifts provides a random fractional shift. The random shifts are again applied to the IEC-X, IEC-Y, and IEC-Z coordinates for the default point of measurement, providing the dosimetric effect due to random interfractional shifts. This is repeated for each fraction in each of the evaluated patient treatment plans. The independent dose calculation here represents the dosimetric effect due to random interfractional shifts. Along the same line, the random shifts reflect the dosimetric effect after systematic correction for intensity guided radiation therapy. The systematic dosimetric variation represents the mean overall effect of dosimetric consequences due to inter-fractional shifts, and the random dosimetric variation represents the specific fractionized effect of dosimetric consequences due to inter-fractional shifts.

Independent dose calculations run for fifty different patient plans including pelvis, prostate, lung, head and neck, and brain anatomic locations. Evaluating different anatomical areas allowed for a more thorough evaluation of the impact of the default, systematic, and random shifts in the accuracy of treatment delivery.

\section{Results - discussion}

The accuracy of the independent dose calculation software was performed without the involvement of any shift. In this step, not only the reference point coordinates were selected, but also the reference dose differences were determined for all patients. The two elements served as the benchmark to the inter-fractional study.

\section{Default inter-fractional shifts}

Starting with the independent dose calculations for treatments incorporating the default shifts, one can see that the data shows good agreement. Shift values were obtained directly from the HT treatment planning station laser coordinates and they were inserted into the in-house software. Figure 1 plots the percent difference values for point dose calculations with the default shift input.

As seen in the graph, the majority of the point dose calculations fell within a $\pm 5 \%$ difference threshold. In fact, $94 \%$ of the data points had a calculated percent difference value within $\pm 5 \%$. Of the fifty patients, only three resulted in a percent dose difference larger than $\pm 5 \%$. The brain, head and neck, and pelvis locations each produced a single occurrence outside this threshold. Of these three instances, however, none have differences larger than $\pm 6 \%$. This suggests good agreement between the treatment planning system calculation and the independent in-house point dose calculator. However, it has to be stated that the data shown are average values from all the fractions that were delivered in each patient. The differences observed are not only a function of patient positioning but also of the shape of the dose distribution applied to each patient. This

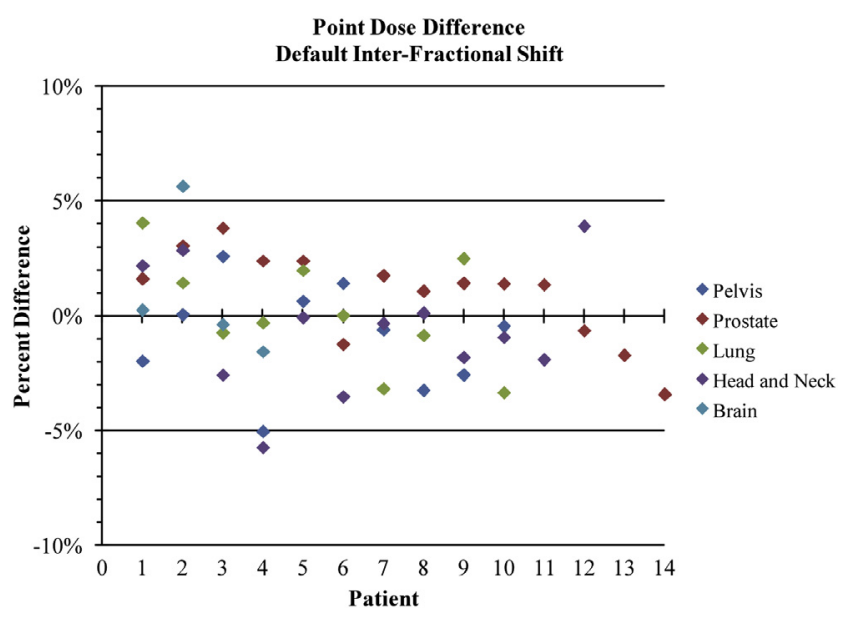

Figure 1. Point dose difference for the default inter-fractional shift in the in-house software.

means that patients belonging to the same cancer site and being characterized by very similar setup shifts may present considerably different dosimetric deviations between the treatment planning system and the in-house software.

The left column of Table 1 provides a quick glance at the general tendencies for the different treatment locations. Percent difference values were averaged across all patients within the specific treatment category for the default shift input calculations. The table clearly shows, that on average, the default shift data from the inhouse software provides a calculation within $\pm 1 \%$ of the treatment planning system calculation. This is a very good agreement.

\section{Systematic inter-fractional shifts}

The systematic shift represents the average raw setup data recorded prior to each treatment fraction. The value is then applied to the default input, creating the systematic shift input. This input adjustment attempts to show, on average, how the point dose calculation is affected should no setup shift be applied. The inhouse software is again used to perform an independent point dose calculation for the corrected shift values. The resulting calculations are graphed in Fig. 2, displaying point dose values minus the systematic shift.

As seen in Fig. 2, correcting for the systematic shift input values leads to point dose calculations slightly less accurate than those seen with the default shift input. Out of the fifty patient examples, a total of three fell outside of the $\pm 5 \%$ window. This means that $94 \%$ of the studied data points had a calculated percent difference value within $\pm 5 \%$ for the systematic shift data. Of these three patients, one is attributed to the pelvis, one to the prostate and one to the lung anatomic sites. Two of the three patients have percent differences below $\pm 10 \%$ (around 8-9\%). The largest percent difference has a value of $-16.9 \%$. Regarding the systematic shifts, an

Table 1

Average percent difference values ( \pm their standard deviation) between the HT treatment planning system and the in-house independent dose calculator for point dose calculations with the default, systematic and random inter-fractional shift input.

\begin{tabular}{lrcr}
\hline Location & Default shift & Systematic shift & Random shift \\
\hline Pelvis & $-0.9 \pm 2.3 \%$ & $-3.0 \pm 5.4 \%$ & $-0.3 \pm 1.5 \%$ \\
Prostate & $1.0 \pm 2.0 \%$ & $2.0 \pm 2.5 \%$ & $-0.2 \pm 1.6 \%$ \\
Lung & $0.2 \pm 2.4 \%$ & $-1.3 \pm 2.9 \%$ & $0.4 \pm 1.9 \%$ \\
Head \& neck & $-0.6 \pm 2.8 \%$ & $-1.0 \pm 1.7 \%$ & $-0.2 \pm 2.0 \%$ \\
Brain & $1.0 \pm 3.2 \%$ & $-1.3 \pm 1.9 \%$ & $1.1 \pm 1.4 \%$ \\
\hline
\end{tabular}




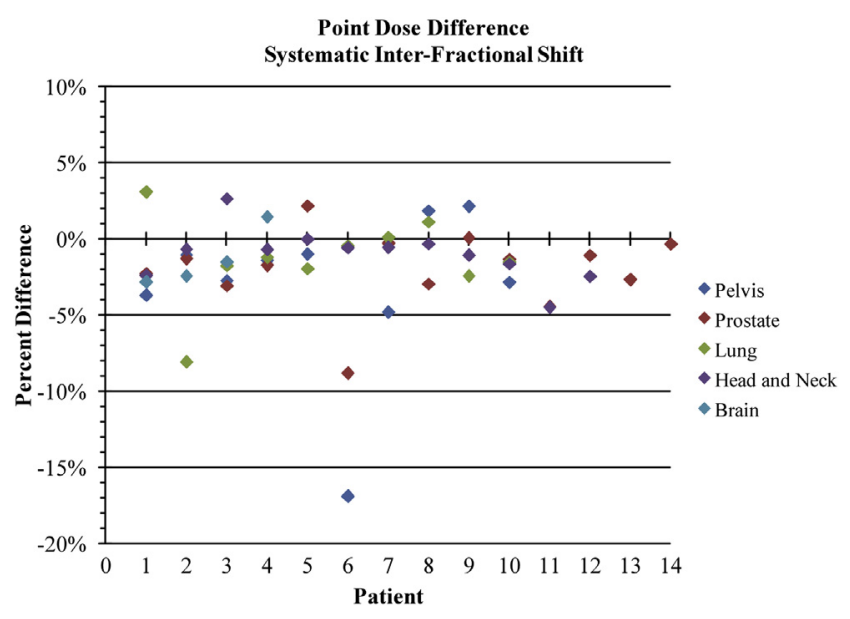

Figure 2. Point dose difference for the systematic inter-fractional shift in the in-house software.

acceptable agreement between the treatment plan and in-house software calculations was observed, overall.

The middle column of Table 1 lists the general tendencies for the different treatment locations with the systematic shift input. Again, percent difference values were averaged over all the patients within each treatment site for the systematic shift input calculations. The table clearly shows, that on average, the default shift data from the in-house software provides a calculation within $\pm 3 \%$ of the treatment planning system calculation. The largest average difference observed is found in the pelvis location category with an average difference of $-3.0 \%$. The remaining treatment location sites all have average percent difference values below $\pm 2 \%$.

For the studied sample of fifty patients, the in-house software has shown to independently calculate point dose to within $\pm 5 \%$ for $94 \%$ of the cases with the systematic shift input. The systematic shift data shows on average, the dose to the selected measurement point if the patient setup shifts had not been applied prior to treatment. While not as accurate as the previous default shift input data, this still shows, on average, good agreement between the treatment planning system and the independent point dose calculations.

\section{Random inter-fractional shifts}

To further explore the shift effect, the in-house software was used to calculate the point dose for each fraction based on the pretreatment shifts recorded in the clinic. This gives an insight about how the point dose calculation would be affected if no treatment shifts were applied. The right column of Table 1 offers an overview of the average fractional shift calculation data. This table displays the percent difference values between the treatment planning system and the in-house independent dose calculation, averaged over all the recorded data fractions within each anatomical site.

This shows that when looking at every fraction for a particular anatomic grouping, the resulting average percent difference is within about $\pm 1 \%$. The largest average percent difference is observed in the brain patients, with a $-1.1 \%$ difference. On the other hand, the smallest average percent difference is observed in the prostate and head \& neck patients, with a $-0.2 \%$ difference.

These average percent difference values are in-line with those observed in the default shift input values, and are better than those seen in the systematic shift input values. Overall, when averaging over all the fractions, for all the patients within a certain anatomic grouping, the resulting percent difference values show very good agreement.
Delving a little further into the data, individual fraction calculations are broken down into counts for the different anatomic groups. Table 2 provides a count of the total number of fraction calculations for each group, the count of fractions with percent difference values greater than $\pm 5 \%$, and the percentage of fractional calculation points with a percent difference that falls within the $\pm 5 \%$ window.

Looking at Table 2, one can see in the second count column that the head \& neck anatomic site has the highest count for calculations with percent difference greater than $\pm 5 \%$, with twenty occurrences. This is four times as many occurrences as seen in any of the other anatomic sites, suggesting that the head \& neck point dose calculations have a lower accuracy than the other anatomic sites.

The ratio of calculations generating a percent difference value within the defined window is lowest for the head \& neck anatomical site, as seen in the final column. Approximately $94 \%$ of the calculations produced a percent difference value within the five percent window. The brain anatomical site had the highest rate (100\%). For the remaining anatomic sites, the percentage of calculations falling within the five percent window was between $97 \%$ and $100 \%$. So, a difference of about three to six percent was observed between the head \& neck and the other anatomical sites for the studied sample.

Inter-fractional point dose calculations have been plotted in respect to the anatomic group (upper diagrams of Figs. 3-7). Point dose percent difference values are plotted for every fraction and patient in each group. Graphs have been scaled to the same size with demarcations placed at the $\pm 5 \%$ gridlines to allow for easy comparison between treatment sites. These graphs plot every fraction calculated with the in-house software.

To provide a more succinct data set, and to present a cleaner figure, the point dose percent differences over all the fractions have been calculated and averaged for each patient (lower diagrams of Figs. 3-7). Standard deviations, providing a measure of how widely the percent difference values are dispersed from the average value, are provided in the form of error bars. This outlines the range of percent difference values one would expect to see at the particular anatomic site.

Pelvis

The upper diagram of Fig. 3 plots the calculation data for the patients with tumors in the pelvis. As can be seen in the graph, the majority of the fractional calculations fall within the $\pm 5 \%$ window. Of the 277 plotted single fraction points, only one does not fall within this threshold. This shows that over 99\% of the random inter-fractional point dose calculations fall within five percent of the default shift values. This shows good agreement for the selected pelvis patient samples, and a fairly minimal dosimetric effect from the random shift component.

Looking into individual patients, it can be seen that Patient 3 produces the sole occurrence outside of the $\pm 5 \%$ window. With a value over $-10 \%$, the point appears to be an irregularity in the Patient

Table 2

Count of total patient fractions contained within each anatomical group, count of fractions resulting in a percent difference value greater than $\pm 5 \%$, and count percentage for the number of points falling within the $\pm 5 \%$ window for the random inter-fractional shift input.

\begin{tabular}{lccl}
\hline Location & Count (\# Fx's) & Count $(> \pm 5 \%)$ & Count percentage $(< \pm 5 \%)$ \\
\hline Pelvis & 277 & 1 & $99.6 \%$ \\
Prostate & 453 & 5 & $98.9 \%$ \\
Lung & 172 & 5 & $97.1 \%$ \\
Head \& neck & 360 & 20 & $94.4 \%$ \\
Brain & 96 & 0 & $100.0 \%$ \\
Total & 1358 & 31 & $97.7 \%$ \\
\hline
\end{tabular}




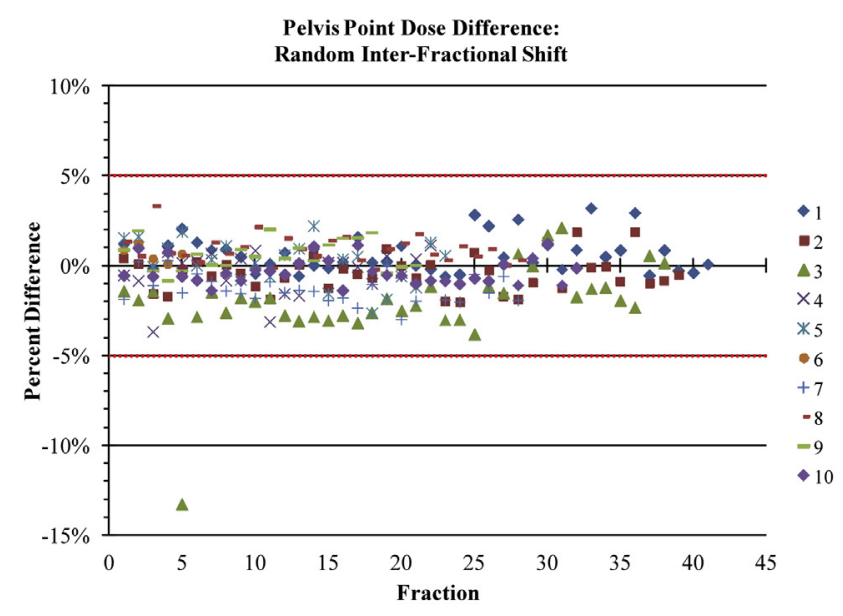

Average Pelvis Percent Difference

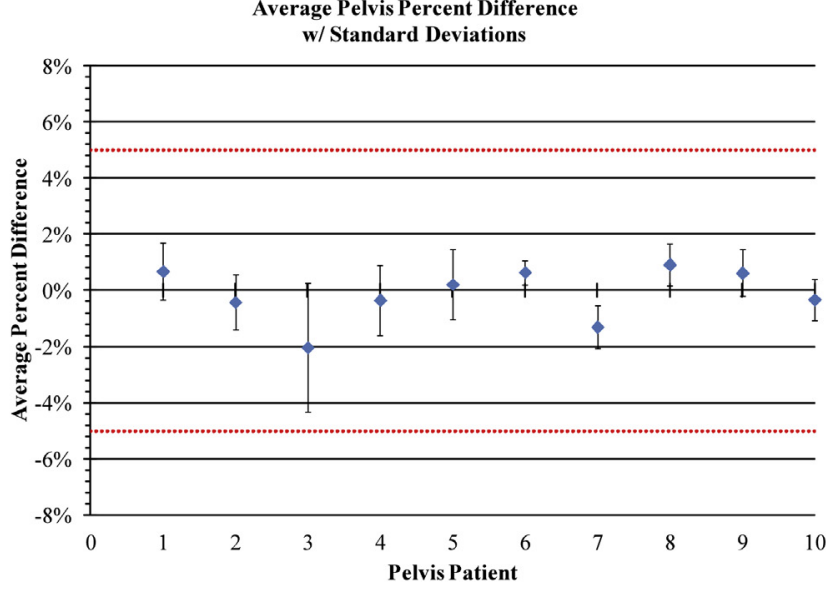

Figure 3. Upper diagram: Point dose difference for the pelvis patient group with the random inter-fractional shift input in the in-house software. All the recorded fractions are calculated and plotted for each patient. Lower diagram: Average percent dose difference for the pelvis patient group with the random inter-fractional shift input in the in-house software. The mean and standard deviation from all the fractions is plotted for each patient.

3 data. An incorrectly recorded shift could explain the difference seen at this particular fraction. Data was recorded for thirty eight fractions for this patient, with only one point valued above $\pm 5 \%$. Averaged over all fractions, the percent differences average to just over $-2 \%$. This is still a small dosimetric effect due to random shifts.

The lower diagram of Fig. 3 displays the average point dose differences for each pelvis patient, with error bars representing the standard deviation for that particular patient data. This gives a general overview of the data for each patient. For the most part, the average percent difference seen for these ten pelvis patients is around or better than $\pm 2 \%$. Looking at the spread of the standard deviations, the data falls within the $\pm 5 \%$ window. As indicated in the previous figure, Patient 3 displays the highest percent difference. This patient has an average percent difference just over $-2 \%$, with a standard deviation span approximately between $0 \%$ and $-4 \%$.

\section{Prostate}

The upper diagram of Fig. 4 plots the calculation data for the prostate patients. Again, the majority of the fractional calculations fall within the $\pm 5 \%$ window. The prostate patient group contains 453 fraction points, with five of them being plotted beyond this window. This shows that almost $99 \%$ of the independent, fractional point dose calculations fall within five percent of the planning system calculation. Again, this shows a good agreement between
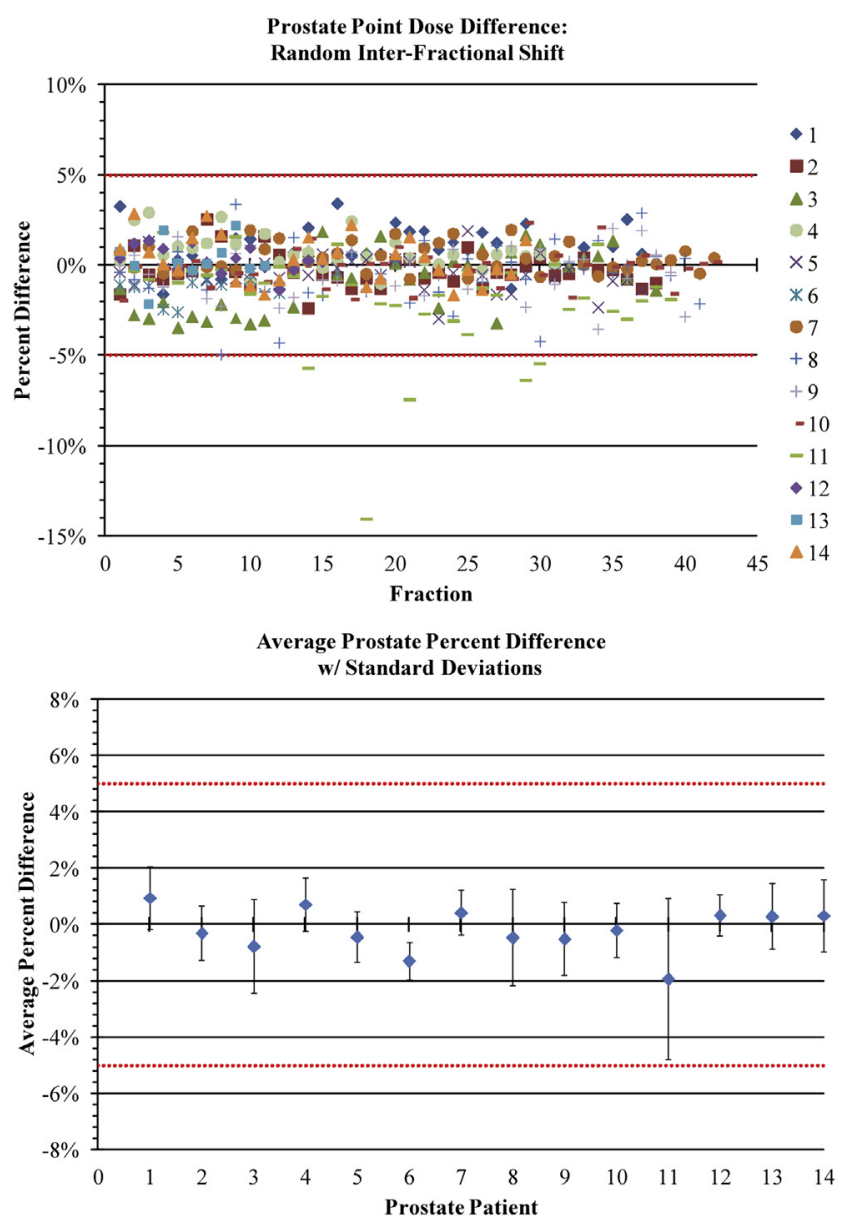

Figure 4. Point dose difference for the prostate patient group. Details as in Fig. 3.

the in-house software and the treatment planning system point dose calculations.

Compared to the pelvis patient group, the data for the prostate patients appear to be more spread out between the five percent demarcations. There is not a particular patient contributing more to the spread, as it was seen in the pelvis group. All the data appears to be evenly dispersed. There does seem to be a tendency for the percent difference values to skew towards either the negative or the positive sides of the axis. Patient 11 appears to be the least accurate of the group. Five of the thirty-nine recorded fractions were beyond the $-5 \%$ difference. A single point fell outside of $-10 \%$, with a difference value of $-14.0 \%$, with the remaining points within $-7 \%$. Most likely, the setup for this particular fraction was incorrect and needed a larger shift correction. It is also possible that this single point was incorrectly recorded.

Looking at the percent differences averaged across all fractions for the prostate cases, the lower diagram of Fig. 4 shows that every patient falls within a $\pm 2 \%$ average window. What's more, the standard deviation error bars all fall within $\mathrm{a} \pm 5 \%$ window. Patient 11 shows the widest data spread, which was seen in the previous figure. Looking at all prostate patients, the averaged random inter-fractional shift calculation displays minimal dosimetric impact on the point dose calculation. All prostate case calculations showed good agreement when averaged over the total treatment falling below a $2 \%$ difference.

Lung

The upper diagram of Fig. 5 plots the calculation data for the lung patients. Again, the majority of the fractional calculations fall 

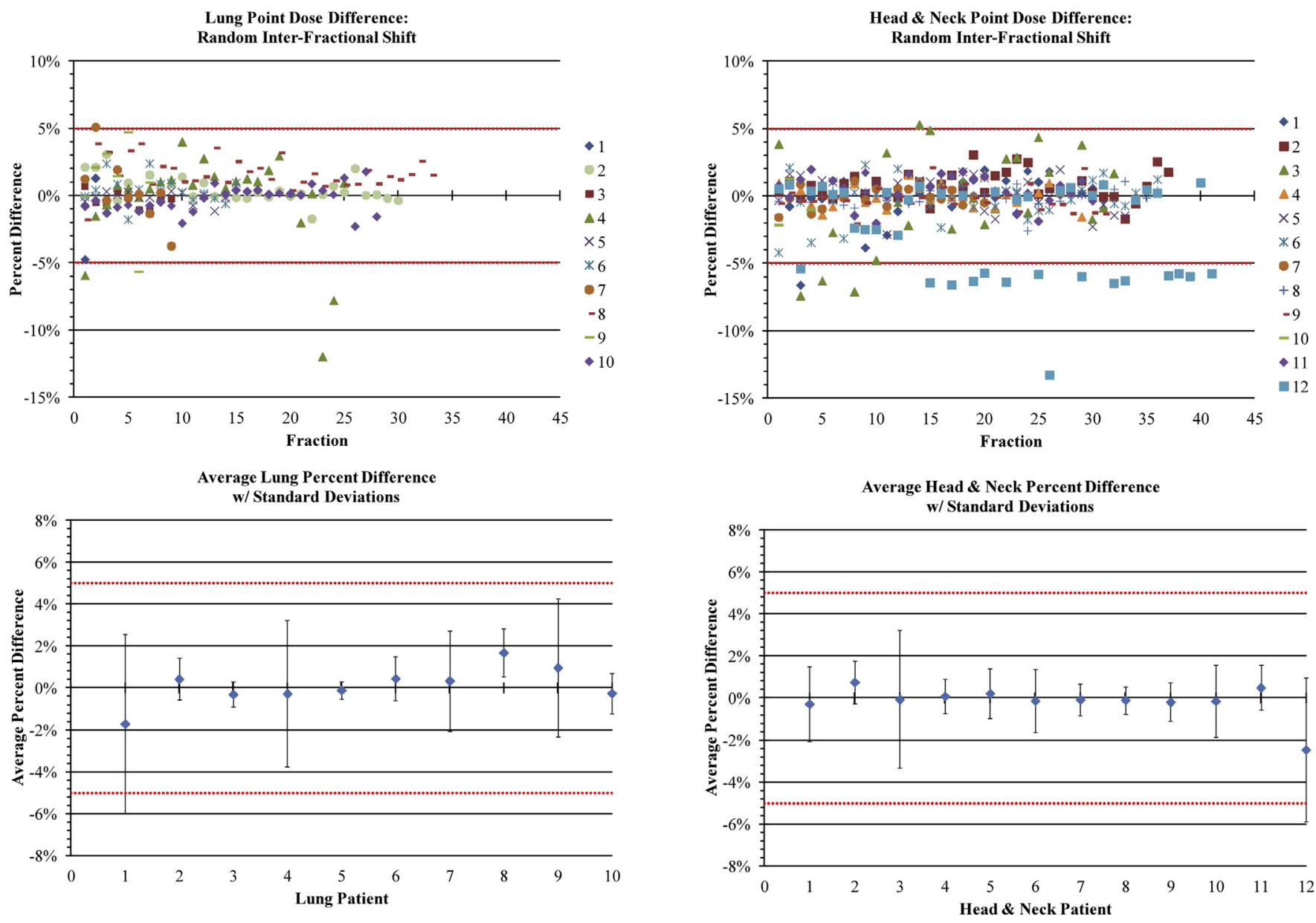

Figure 5. Point dose difference for the lung patient group. Details as in Fig. 3.

within the $\pm 5 \%$ window. The lung patient group contains 172 fraction points, with five of them being plotted beyond this window. This shows that about $97 \%$ of the independent, fractional point dose calculations fall within five percent of the planning system calculation. Again, this shows good agreement between the in-house software and the TPS point dose calculations.

The lung patient calculation plots appear to be spread out quite evenly between the positive and negative sides of the axis. There is only a single data point whose percent difference is beyond $-10 \%$. Three patients produce points with a percent difference greater than $\pm 5 \%$. Only Patient 4 has multiple points beyond $5 \%$. For the rest of the patients this occurs only during a single fraction, with the remaining fractions having percent difference values within the five percent window.

The lower diagram of Fig. 5 displays the average value for the inter-fractional shift calculations for each patient in the lung group. This plot shows that again, possible outliers viewed in the individual fraction calculations are averaged out when looking across the entire treatment. The mean percent difference values for all the patients fall within $\pm 2 \%$. In most of the cases, the standard deviations are also contained within the $\pm 5 \%$ error window. The somewhat large standard deviation values for four of the patients indicate a spread in the data of the individual fraction calculations.

\section{Head \& neck}

The upper diagram of Fig. 6 plots the calculation data for the head and neck patients. This patient group contains 360 fraction points, with twenty of them being plotted beyond the five percent
Figure 6. Point dose difference for the head and neck patient group. Details as in Fig. 3.

window. Therefore, $94.4 \%$ of the random fractional point dose calculations fall within five percent of the planning system calculation. Even though this result shows good agreement between the inhouse and the TPS point dose calculations, it is the lowest percentage among the five anatomic groups.

Data is again evenly distributed throughout the five percent window. Similar to the pelvis group, there is a single patient whose dose difference values are consistently higher than the others. About a third of the inter-fractional points for Patient 12 are consistently plotted just beyond $-5 \%$, with fifteen of the forty-one fractions producing a percent difference greater than $5 \%$. Only a single point is beyond $-10 \%$, with most being calculated at a $-5 \%$ to $-7 \%$ difference.

Possibly due to a location near a steep dose gradient, the point dose of this particular plan is not reproduced during treatment delivery as accurately as those of the other patients.

Two other patients make up the other outlying points, with Patient 1 having a single point and Patient 3 with 4 points. The percent differences beyond the $5 \%$ value drift towards the negative side. Patient 3 produces three values near $-6 \%$ and another near $5 \%$. Patient 1 has a single value near seven percent which appears to be more of an outlier.

The large presence of heterogeneity in the head and neck cases is one possible reason for the observed difference. The heterogeneity presence makes it more difficult to accurately calculate dose, and leads to an increased likelihood of a point being placed at or near a steep dose gradient. This will accentuate the dose differences should the shifts be off. 

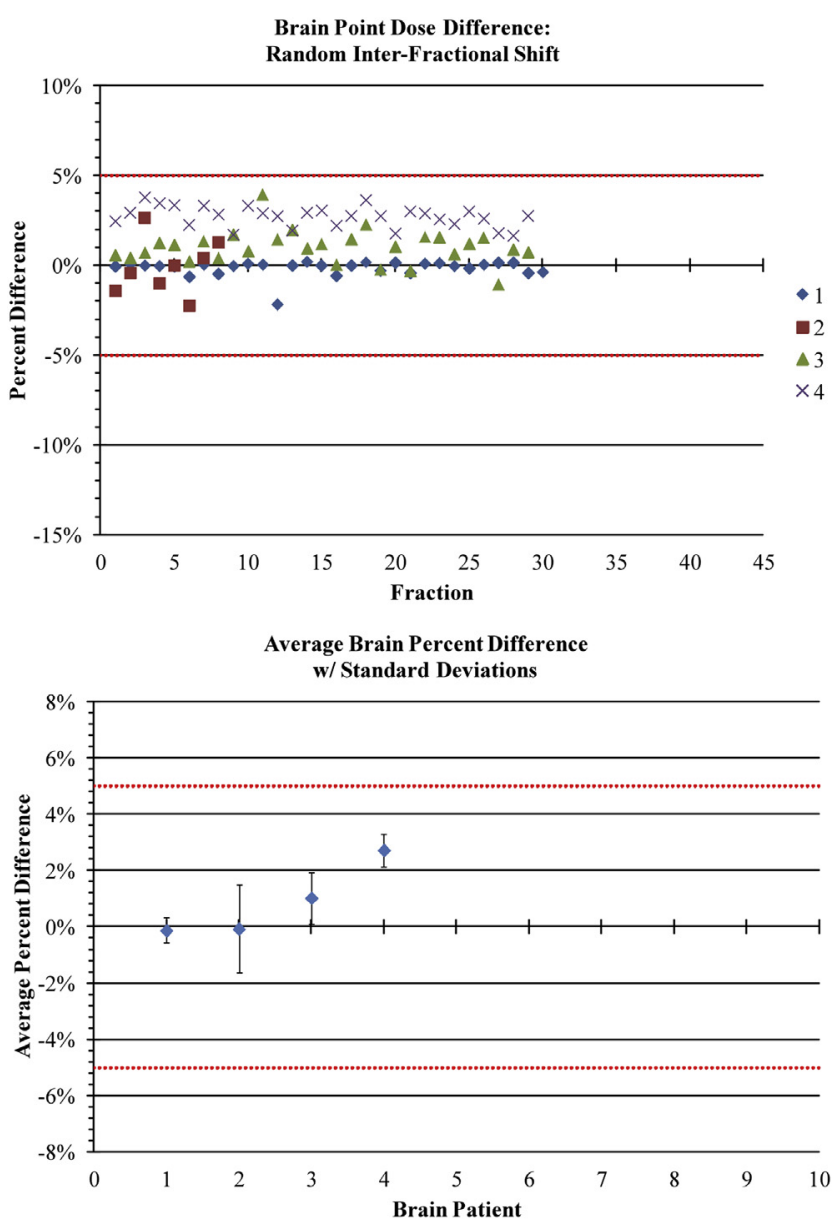

Figure 7. Point dose difference for the brain patient group. Details as in Fig. 3.

Again, the average values displayed in the lower diagram of Fig. 6 reinforce the conclusions drawn from the individual fraction calculations of the previous anatomical sites. The mean percent difference values for the head and neck patients all fall within about two percent. Patient 12, which displayed the largest number of outlying points, has an average value around $-2 \%$, but has a larger data spread. The head and neck patients continue to show minimal dosimetric effect for the random inter-fractional shifts.

\section{Brain}

The upper diagram of Fig. 7 plots the calculation data for the sampled brain patients. The small patient sampling is due to the number of treatments for this particular site in this clinic. This patient group contains 96 fraction points, the fewest among the five anatomic groups, with none of them being plotted beyond the five percent window. This indicates that all of the random fractional point dose calculations fall within five percent of the planning system calculation. Again, this is good agreement between the inhouse software and the treatment planning system point dose calculations.

Patient 4 is the least accurate of this group, with data points hovering around the three percent demarcation. In fact, all other calculated random values hover around two percent or better. Patient 1 shows almost no random shift effect while Patient 2 displays the largest spread.

The lower diagram of Fig. 7 reiterates the data seen in the individual fraction calculations. As expected, all mean values for the random inter-fractional calculations are near, or within, two percent, and have relatively little data spread. The samples all display good point dose calculation agreement.

\section{Overall patient summary}

Extending the data analysis, Table 3 provides the average percent difference values for each patient, separately. The number of studied patient examples was selected based on commonality. Prostate treatments are the most common type for the HT modality at this clinic, which is the reason for selecting a larger number of patients. Additional head \& neck cases were studied to supplement the small number of brain cases available for this study.

As it can be seen, none of the fifty patients produced an average value above the boundary of $5 \%$. The average value over all the fractions for each of the patients was within three percent, with most of the patients being even lower than that. This patient by patient breakdown shows that over the course of a treatment, the random inter-fractional point dose calculations typically average out to within three percent of the default shift values. This suggests that the dosimetric impact due to random inter-fractional shifts is not significant.

From Table 3, it can be seen that the lung cancer site is the most susceptible site to dosimetric differences between the treatment planning and the in-house software calculations. Although the absolute presentation may show that the head \& neck grouping has a lower percentage of fractions with a dose difference between the calculations of the treatment planning and those of the in-house software $<5 \%$ (Table 2 ), most of them stem from a single patient (Patient 12). Table 3 shows a more clear picture of the variation of the results among the different anatomical groupings. So, it shows that the Lung grouping has 3 patients out of 10 with average fractional differences over $1 \%$ (30\%), whereas the head \& neck grouping has only one out of 12 patients (8.3\%).

From Figs. 3-7, it can be observed that the Pelvis, Prostate, head \& neck and Brain anatomical groupings show small inter-fractional variations, with the exception of one patient (outlier) per grouping. The largest inter-fractional variation is observed for the Lung grouping both in magnitude and in the proportion of patients showing more extensive variations. However, it must be pointed out that the results depend on both the shape of the applied dose distribution as well as the position of the reference calculation point within the patient. So, in such kind of studies, patient setup uncertainties may play a smaller role in the accuracy by which the dose at a point is calculated than the placement of that point in a highly conformal dose distribution. In the different cancer sites examined in the present study, the involvement of OARs and their proximity to the irradiated target varies considerably. This fact has a

Table 3

Lists the average fractional percent difference values for each anatomical group per patient.

\begin{tabular}{llllll}
\hline \multicolumn{6}{l}{ Average fractional differences per patient (\%) } \\
\hline Patient & Pelvis & Prostate & Lung & Head \& neck & Brain \\
\hline 1 & $0.7 \%$ & $1.0 \%$ & $-1.7 \%$ & $-0.3 \%$ & $-0.1 \%$ \\
2 & $-0.4 \%$ & $-0.3 \%$ & $0.4 \%$ & $0.8 \%$ & $-0.1 \%$ \\
3 & $-2.0 \%$ & $-0.8 \%$ & $-0.3 \%$ & $-0.1 \%$ & $1.0 \%$ \\
4 & $-0.3 \%$ & $0.7 \%$ & $-0.3 \%$ & $0.1 \%$ & $2.7 \%$ \\
5 & $0.2 \%$ & $-0.4 \%$ & $-0.1 \%$ & $0.2 \%$ & \\
6 & $0.6 \%$ & $-1.3 \%$ & $0.5 \%$ & $-0.1 \%$ & \\
7 & $-1.3 \%$ & $0.4 \%$ & $0.3 \%$ & $-0.1 \%$ & \\
8 & $0.9 \%$ & $-0.5 \%$ & $1.7 \%$ & $-0.1 \%$ & \\
9 & $0.6 \%$ & $-0.5 \%$ & $1.0 \%$ & $-0.2 \%$ & \\
10 & $-0.3 \%$ & $-0.2 \%$ & $-0.3 \%$ & $-0.1 \%$ & \\
11 & & $-1.9 \%$ & & $0.5 \%$ & \\
12 & $0.3 \%$ & & $-2.5 \%$ & \\
13 & $0.3 \%$ & & & \\
14 & $0.3 \%$ & & & \\
\hline
\end{tabular}


consequence the production of dose distributions whose conformity also varies considerably among the patients of the different anatomical groupings as well as among the patient of the same grouping. Furthermore, the placement of the reference dose calculation point was made without any a priori knowledge of the calculations of the in-house software. This means that by placing the reference dose calculation point at a region where dose is more homogenous, away from steep dose fall-off edges would lead to a much close agreement between the calculations of the treatment planning system and those of the in-house software for all the examined clinical cases.

In the recent years, there is an increasing interest for tools that could perform independent dose calculations for IMRT and other highly conformal radiation modalities as HT. However, the publications that describe adequately the accuracy of their tools and have acquired adequate experience in using them are scarce $[27,35,36]$. This is because in order to develop a highly accurate tool for independent dose calculation, more general (semi-analytical) models than the traditionally used factor-based models must be implemented. Additionally, these models should be independent of the TPS and should be based on physical effects which should be accurately described by an independent set of input data providing also an estimation of the overall uncertainty in the dose calculation [37].

The presented software provides independent dose calculations based on the commissioned dosimetric data from a given tomotherapy unit and it retrieves dynamic information such as the MLC movement, gantry projections and couch translations with specified pitch from the treatment plan of each examined patient. The dosimetric results of the study were derived based on the assumption that patients were rigid bodies. Since this work studied the inter-fractional nature and impact to the dose distribution, rigid body assumption is a simple way for the incorporation of the setup shift on coordinates, which may introduce calculation inaccuracy due to possible deformable activities inside the patient. Consequently, the presence of intra-fractional motions is another factor that should be investigated and accounted for by the independent dose calculation software.

In the present study, the dosimetric variations were classified into systematic and random. The systematic dosimetric variations were derived from the systematic shift of the individual patients, whereas the random dosimetric variations were derived from the random shifts after the systematic variation for each treatment fraction has been accounted for. Due to variations in patient status and anatomy, individual patients may have their own characteristics in daily setup. Consequently, an individual registration may not be normally derived from a population of observations. Furthermore, specific studies have shown different features in systematic dosimetric variations between groups with different internal motions and common agreement within each group. These findings indicate that the systematic shift impact is relatively treatment site related. Consequently, different treatment sites may exhibit different setup shifts and require different registration techniques and tolerances.

Different characteristics from systematic and random interfractional shifts show different dosimetric impacts on patient specific and treatment site specific plans. For example, it can be noticed that head \& neck and brain patients, who have the small daily setup shifts due to immobilization equipments, may have large dosimetric variations. This is because the treatment plans on these two sites focus on superficial or small regions and consequently they are more sensitive to position shifts. Although there is a number of studies, which have examined the effect of patient setup shifts in conformal and IMRT techniques for different treatment sites [33,38-43], most patient setup shift studies in Helical
Tomotherapy focused on the MVCT itself in relation to novel registration strategies [14-20]. Although a few studies presented results on dosimetric consequences with the MVCT setup shifts for patients with prostate cancer [21], head \& neck cancer [44] and breast cancer [22], the dose calculations were repeated using the same treatment planning system as the original plan.

Among the limitations of the presented software is the point dose comparison only and random sampling of the point inside the target. In the future, those limitations may be overcome by comparing multi-point calculations from PTV or surrounding sensitive structures and having the possibility of comparing two dimensional or three-dimensional dose distributions. However, the current method has provided a good way to calculate the dose independently for tomotherapy modality in a short time.

\section{Conclusion}

It has been shown that the in-house software is a capable platform for independent point dose validation for the Helical Tomotherapy modality. For the selected groups of patients, the results showed good agreement between the clinical treatment planning system calculations and the in-house software independent point dose calculations. Furthermore, the effect that patient shifts played on the accuracy of treatment delivery was evaluated. Calculations were performed for the default treatment planning system shifts, for the systematic inter-fractional shifts, as well as for the random inter-fractional shifts. The results from the treatment planning system and those from the in-house software typically showed good agreement for all the three calculated phases. Association of the calculation point position with the shape of the applied dose distribution can improve even more the agreement of the calculations from the treatment planning system and the inhouse software. Prospective goals entail further development of this independent dose validation tool. Further advancement of heterogeneity correction could potentially improve the calculation accuracy. Also, the development of an extra module for the calculation of the dose distribution throughout a volume needs to be addressed.

\section{References}

[1] Mackie TR, Kapatoes J, Ruchala K, Lu WG, Wu C, et al. Image guidance for precise conformal radiotherapy. Int J Radiat Oncol 2003;56:89-105.

[2] Perkins CL, Fox T, Elder E, Kooby DA, Staley CA, et al. Image-guided radiation therapy (IGRT) in gastrointestinal tumors. JOP 2006;7:372-81.

[3] Sheng K, Chow J, Hunter G, Larner JM, Read PW. Is daily CT image guidance necessary for nasal cavity and nasopharyngeal radiotherapy: an investigation based on helical tomotherapy. J Appl Clin Med Phys 2008;9:2686.

[4] Stutzel J, Oelfke U, Nill S. A quantitative image quality comparison of four different image guided radiotherapy devices. Radiother Oncol 2008;86:20-4.

[5] Mackie TR. History of tomotherapy. Phys Med Biol 2006;51:R427-53.

[6] Keall PJ, Mageras GS, Balter JM, Emery RS, Forster KM, et al. The management of respiratory motion in radiation oncology report of AAPM Task Group 76. Med Phys 2006;33:3874-900.

[7] Sawant A, Venkat R, Srivastava V, Carlson D, Povzner S, et al. Management of three-dimensional intrafraction motion through real-time DMLC tracking. Med Phys 2008;35:2050-61.

[8] Lefkopoulos D, Ferreira I, Isambert A, Le Pechoux C, Mornex F. Present and future of the Image Guided Radiotherapy (IGRT) and its applications in lung cancer treatment. Cancer Radiotherapie 2007;11:23-31.

[9] Su FC, Shi CY, Crownover R, Swanson G, Papanikolaou N. Dosimetric impacts of gantry angle misalignment on prostate cancer treatment using helical tomotherapy. Technol Cancer Res Treat 2008;7:287-93.

[10] Low DA, Zhu XR, Purdy JA, Soderstrom S. The influence of angular misalignment on fixed-portal intensity modulated radiation therapy. Med Phys 1997;24:1123-39.

[11] Xing L, Lin ZX, Donaldson SS, Le QT, Tate D, et al. Dosimetric effects of patient displacement and collimator and gantry angle misalignment on intensity modulated radiation therapy. Radiother Oncol 2000;56:97-108.

[12] Lu W, Chen M, Ruchala KJ, Chen Q Langen KM, et al. Real-time motionadaptive-optimization (MAO) in TomoTherapy. Phys Med Biol 2009;54: 4373-98. 
[13] Ngwa W, Meeks SL, Kupelian PA, Schnarr E, Langen KM. Validation of a computational method for assessing the impact of intra-fraction motion on helical tomotherapy plans. Phys Med Biol 2009;54:6611-21.

[14] Yan D, Lockman D, Martinez A, Wong J, Brabbins D, et al. Computed tomography guided management of interfractional patient variation. Semin Radiat Oncol 2005;15:168-79.

[15] Broggi S, Di Muzio N, Fiorino C, Cozzarini C, Cattaneo GM, et al. Prostate tracking through daily MVCT-guided tomotherapy: reduced motion by careful emptying of the rectum. Radiother Oncol 2007;84:393.

[16] Broggi S, Cozzarini C, Fiorino C, Maggiulli E, Alongi F, et al. Modeling set-up error by daily MVCT for prostate adjuvant treatment delivered in 20 fractions: implications for the assessment of the optimal correction strategies. Radiother Oncol 2009;93:246-52.

[17] Lee C, Langen KM, Lu W, Haimerl J, Schnarr E, et al. Evaluation of geometric changes of parotid glands during head and neck cancer radiotherapy using daily MVCT and automatic deformable registration. Radiother Oncol 2008;89: 81-8.

[18] Lin L, Shi CY, Eng T, Swanson G, Fuss M, et al. Evaluation of interfractional setup shifts for site-specific helical tomotherapy treatments. Technol Cancer Res Treat 2009;8:115-22.

[19] Ryan D, Rivest C, Riauka TA, Murtha AD, Fallone BG. Prostate positioning errors associated with two automatic registration based image guidance strategies. J Appl Clin Med Phys 2009;10:165-76.

[20] Li XA, Qi XS, Pitterle M, Kalakota K, Mueller K, et al. Inter-fractional variations in patient setup and anatomic change assessed by daily $\mathrm{CT}$ from helical tomotherapy. Int J Radiat Oncol Biol Phys 2007;68:581-91.

[21] Kalz J, Sterzing F, Schubert K, Sroka-Perez G, Debus J, et al. Dosimetric comparison of image guidance by megavoltage computed tomography versus bone alignment for prostate cancer radiotherapy. Strahlenther Onkol 2009;185:241-7.

[22] Goddu SM, Yaddanapudi S, Pechenaya OL, Chaudhari SR, Klein EE, et al. Dosimetric consequences of uncorrected setup errors in helical Tomotherapy treatments of breast-cancer patients. Radiother Oncol 2009;93:64-70.

[23] Mavroidis P, Stathakis S, Gutierrez A, Esquivel C, Shi C, Papanikolaou N. Expected clinical impact of the differences between planned and delivered dose distributions in Helical Tomotherapy for treating head and neck cancer using Helical Megavoltage CT Images. J Appl Clin Med Phys 2009;10: 2969-89.

[24] Mavroidis P, Su F, Giantsoudi D, Stathakis S, Komisopoulos G, Shi C, et al. Radiobiological and dosimetric analysis of daily megavoltage $\mathrm{CT}$ registration techniques on adaptive radiotherapy with Helical Tomotherapy. Technol Cancer Res Treat 2011;10:1-13.

[25] Kutcher GJ, Coia L, Gillin M, Hanson WF, Leibel S, Morton RJ, et al. Comprehensive QA for radiation oncology: report of AAPM radiation therapy Committee Task Group 40. Med Phys 1994;21:581-618.

[26] Karlsson M, Ahnesjö A, Georg D, Nyholm T, Olofsson J. Independent dose calculations concepts and models: ESTRO booklet 10. Brussels, Belgium: European Society for Radiotherapy and Oncology; 2010.

[27] Georg D, Stock M, Kroupa B, Olofsson J, Nyholm T, Ahnesjö A, et al. Patientspecific IMRT verification using independent fluence-based dose calculation software: experimental benchmarking and initial clinical experience. Phys Med Biol 2007;52(16):4981-92.
[28] Papanikolaou N, He W, Vazquez QLA, Gutierrez A, Stathakis S, Alkhatib H, et al. MU-Tomo: independent dose validation software for Helical Tomotherapy. J Cancer Sci Ther 2010;2:145-52.

[29] Mukumoto N, Tsujii K, Saito S, Yasunaga M, Takegawa H, Yamamoto T, et al. A preliminary study of in-house Monte Carlo simulations: an integrated Monte Carlo verification system. Int J Radiat Oncol Biol Phys 2009;75:571-9.

[30] Pisaturo O, Moeckli R, Mirimanoff RO, Bochud FO. A Monte Carlo-based procedure for independent monitor unit calculation in IMRT treatment plans. Phys Med Biol 2009;54:4299-310.

[31] Tsai JS, Engler MJ, Liu J. Quasi-independent monitor unit calculation for intensity modulated sequential tomotherapy. J Appl Clin Med Phys 2002;3:135-53.

[32] Ma CM, Li J, Pawlicki T. In: Proceedings of the XIVth International Conference on the Use of Computers in Radiation Therapy (ICCR), Seoul (Korea); 2004.

[33] Fu WH, Yang Y, Li X, Heron DE, Huq MS, et al. Dosimetric effects of patient rotational setup errors on prostate IMRT treatments. Phys Med Biol 2006;51: 5321-31.

[34] He Weihong, Luis A, Vazquez Q Dzintars Eric, Papanikolaou Nikos, Shi Chengyu. Assessing the dosimetric consequence of inter-fractional setup shifts on helical TomoTherapy plans with independent dose calculation. J Cancer Sci Ther 2010;2:136-44.

[35] Georg D, Nyholm T, Olofsson J, Kjaer-Kristoffersen F, Schnekenburger B, Winkler P, et al. Clinical evaluation of monitor unit software and the application of action levels. Radiother Oncol 2007;85:306-15.

[36] Linthout N, Verellen D, Van Acker S, Storme G. A simple theoretical verification of monitor unit calculation for intensity modulated beams using dynamic mini-multileaf collimation. Radiother Oncol 2004;71:235-41.

[37] Olofsson J, Nyholm T, Georg D, Ahnesjö A, Karlsson M. Evaluation of uncertainty predictions and dose output for model-based dose calculations for megavoltage photon beams. Med Phys 2006;33:2548-56.

[38] Zhou J, Uhl B, Dewit K, Young M, Taylor B, et al. Analysis of daily setup variation with tomotherapy megavoltage computed tomography. Med Dosim 2010;35:31-7.

[39] Fiorino C, Di Muzio N, Broggi S, Cozzarini C, Maggiulli E, et al. Evidence of limited motion of the prostate by carefully emptying the rectum as assessed by daily MVCT image guidance with helical tomotherapy. Int J Radiat Oncol 2007;71:611-7.

[40] Siebers JV, Keall PJ, Wu QW, Williamson JF, Schmidt-Ullrich RK. Effect of patient setup errors on simultaneously integrated boost head and neck IMRT treatment plans. Int J Radiat Oncol Biol Phys 2005;63:422-33.

[41] Manning M, Wu Q, Cardinale RM, Mohan R, Lauve AD, et al. The effect of setup uncertainty on normal tissue sparing with IMRT for head and neck cancer. Int J Radiat Oncol Biol Phys 2001;51:1400-9.

[42] Landoni V, Saracino B, Marzi S, Gallucci M, Petrongari MG, et al. A study of the effect of setup errors and organ motion on prostate cancer treatment with IMRT. Int J Radiat Oncol Biol Phys 2006;65:587-94.

[43] Balter JM, Brock KK, Lam KL, Tatro D, Dawson LA, et al. Evaluating the influence of setup uncertainties on treatment planning for focal liver tumors. Int J Radiat Oncol Biol Phys 2005;63:610-4.

[44] Mavroidis P, Stathakis S, Gutierrez A, Esquivel C, Shi C, Papanikolaou N. Expected clinical impact of the differences between planned and delivered dose distributions in helical tomotherapy for treating head and neck cancer using helical megavoltage CT images. J Appl Clin Med Phys 2009;10:125-39. 